\title{
Optimal Droop Control for Voltage Source Converters in Islanded Microgrids
}

\author{
Jordi Pegueroles-Queralt* Fernando Bianchi * \\ Oriol Gomis-Bellmunt ${ }^{*, \dagger}$ \\ * Institut de Recerca en Energia de Catalunya, IREC, Barcelona, \\ 08930 Spain (e-mail: jpegueroles,fbianchi,ogomis@irec.cat). \\ † Centre d'Innovació Tecnològica en Convertidors Estàtics $i$ \\ Accionaments (CITCEA-UPC) \\ Universitat Politècnica de Catalunya, \\ Av. Diagonal 647, Pl. 2, 08028 Barcelona, Spain
}

\begin{abstract}
:
A new procedure for the design of the droop control for voltage source converters (VSC) in islanded microgrid is presented. The droop control is commonly used to achieve the power balance in power system. It consists in a simple proportional control and the selection of its gains are commonly based only on power balance criteria. However, some values of the gains may cause poor damped responses and even the instability of the system. In this paper, it is proposed a new droop control based on the parametrization of the $H_{\infty}$ controller which permits to decouple the stability problem from the power balance. The application of the new controller is illustrated by simulation.
\end{abstract}

Keywords: Microgrid, optimal control, isolated networks, voltage source converters, frequency control, power systems.

\section{INTRODUCTION}

To meet the "20-20-20" target of the European Union (EU Commission, 2010) the future power system will have a high penetration of renewable energy sources (RES). This scenario of high RES penetration will demand a change in the current electricity generation landscape to the so-called smart grid (EU Commission, 2006). That is, a scenario where the power provided by the generation sources and the power demanded by the consumers are regulated in order to achieve a better use of the available energy. Before reaching a global smart grid, the microgrid is a solution to form small scale smart power systems, connected to the utility by a single point (Lasseter, 2002).

A microgrid is a smart integration of generation and energy storage units with a cluster of loads, some of them controllable, which can work as an isolated local controllable power system by disconnecting from the utility at the point of common coupling (PCC) (see Fig. 1). Microgrids have two different operation modes: grid-connected mode acting as a controlled power source and islanded mode supplying loads during grid disturbances or intended islandings. The objective of the microgrid is to obtain accurate load sharing while assuring stability and good regulation of the voltage and frequency (IEEE Standards Coordinating Committee 21, 2011). For the scope of this paper, microgrids are considered as illustrated in Fig. 1. Each generation unit is composed of a RES together with a voltage source converter (VSC). The latter serve as an interface between the variable renewable power sources and the grid.
In the last few years much effort has been put on the research of many aspects, from architecture to control strategies. Many of these developments are being implemented in test beds spread across the world. Among them are: CERTS microgrid project in U.S., Hachinohe project in Japan (Agrawal and Mittal, 2011), and others like IREC's microgrid (Roman-Barri et al., 2010).

The control strategies for microgrids can be based on centralized or decentralized paradigms. The first ones like the master-slave or the multi-master scheme as presented

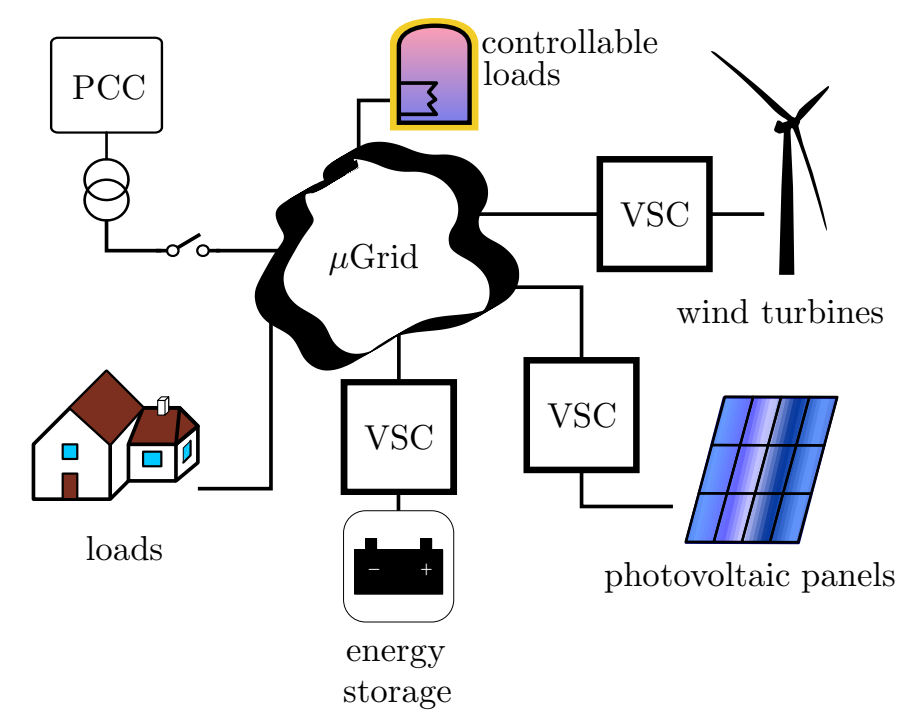

Fig. 1. Islanded microgrid scheme 
by Lopes and Moreira (2006) are able to achieve a better power balance but they need communications. In decentralized schemes, the power sharing is achieved by controllers using only local measurements. Independently of the paradigm used, a robust microgrid should include some low level control that does not rely on communications or on a master-slave scheme. This decentralized low-level control is commonly referred to as droop control (Guerrero et al., 2004). In these strategies, each unit has the same responsibility to keep the frequency and the voltage stable (Pogaku et al., 2007). Droop control is basically a proportional control, therefore it is difficult to find a compromise between the error needed for power sharing and stability. This article is focused on the droop control of microgrids, in particular, it presents a novel design procedure based $H_{\infty}$ optimal control that permits to decouple the power balance from the stabilization problem.

\section{MODEL DESCRIPTION}

In order to design the droop control, it is necessary to characterize the effect of the droop on the power flow in the microgrid. To this end, the microgrid sketched in Fig. 1 is modelled with a equivalent impedance $Z \angle \theta=R+j \omega L$ and a balanced three phase voltage $U_{2} \angle \delta$, as shown in Fig. 2. Each VSC in the microgrid is equipped with a $L_{f} C_{f} L_{x}$ filter to smooth the current ripple caused by the switching of the power devices. Only local measurements of current $I_{0}$ and $I$, and voltage $U_{1}$ are the required control inputs for the VSC. For the design and analysis of the droop controller, the RES is modelled as a constant voltage source.

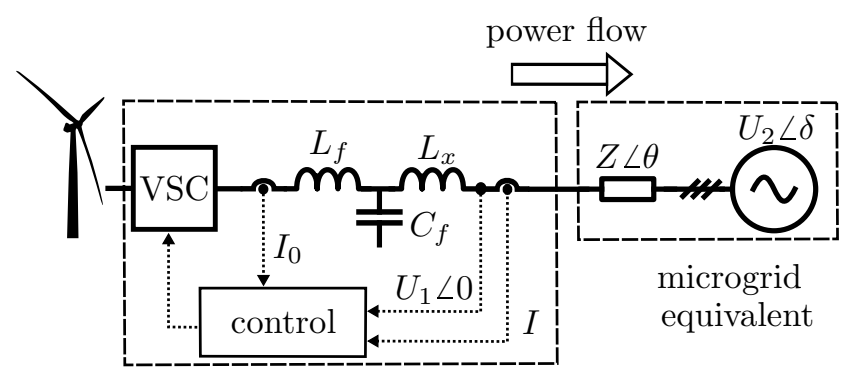

Fig. 2. Equivalent microgrid scheme

The power flow is originated by a change in angle $\delta$ or a voltage difference between $U_{1}$ and $U_{2}$. From the phasor diagram in Fig. 3, the voltage across an impedance with inductance $L$ and resistance $R$ is given by

$$
U_{1 a b c}(t)-U_{2 a b c}(t)=R I_{a b c}(t)+L \frac{d}{d t} I_{a b c}(t),
$$

where $U_{1 a b c}(t)$ is the VSC output voltage, $U_{2 a b c}(t)$ is the equivalent microgrid voltage, and $I_{a b c}(t)$ is the current flowing through the equivalent impedance, all variables are in the $a b c$ frame.

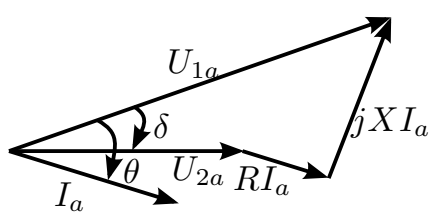

Fig. 3. Phasor diagram of the generation units power flow

Applying the Park's transformation to equation (1), it is obtained

$$
\begin{aligned}
& L \frac{d}{d t} i_{d}+R i_{d}-\omega L i_{q}=u_{1 d}-u_{2 d} \\
& L \frac{d}{d t} i_{q}+R i_{q}+\omega L i_{d}=u_{1 q}-u_{2 q}
\end{aligned}
$$

The Park's transformation projects the magnitudes to a frame rotating at $\omega$ synchronized to the $d$ axis, such that component $u_{1 q}=0$. The equivalent impedance $Z$ causes a deviation angle $\delta$ between $U_{1}$ and $U_{2}$. The voltage $u_{2 d q}$ can be expressed as

$$
\begin{aligned}
& u_{2 d}=U_{2 a} \cos \delta \\
& u_{2 q}=U_{2 a} \sin \delta .
\end{aligned}
$$

Rearranging (2), (3), (4) and (5)

$$
\frac{d}{d t}\left[\begin{array}{l}
i_{d} \\
i_{q}
\end{array}\right]=\left[\begin{array}{cc}
-\frac{R}{L} & \omega \\
-\omega & -\frac{R}{L}
\end{array}\right]\left[\begin{array}{c}
i_{d} \\
i_{q}
\end{array}\right]+\left[\begin{array}{c}
\frac{U_{1}-U_{2 a} \cos \delta}{L} \\
-\frac{U_{2 a} \sin \delta}{L}
\end{array}\right] .
$$

Applying the Instantaneous power theory (Akagi et al., 2007 ), the active and reactive power flowing out of the $\mathrm{VSC}$ can be expressed as

$$
\begin{aligned}
& P=\frac{3}{2}\left(u_{1 d} i_{d}+u_{1 q} i_{q}\right)=\frac{3}{2} u_{1 d} i_{d}, \\
& Q=\frac{3}{2}\left(u_{1 d} i_{q}-u_{1 q} i_{d}\right)=\frac{3}{2} u_{1 d} i_{q},
\end{aligned}
$$

using $u_{1 q}=0$.

After substituting (6) in the expressions of $P$ and $Q$, and linearizing around the operating conditions, equations (7) and (8) become the linear model (9)-(10), where^denotes a small deviation respect to operating conditions, and the subindexes $o$ denote the operating point values, and $U_{o}$ is the nominal voltage of the microgrid.

Notice that equal results can be obtained using the dynamic phasor theory as described by Venkatasubramanian et al. (1995). Dynamic phasors are commonly used for the analysis of subsynchronous resonance and the interaction of network and load dynamics in power systems (Allen and Ilic, 2000).

$$
\begin{gathered}
\frac{d}{d t}\left[\begin{array}{l}
\hat{i}_{d} \\
\hat{i}_{q}
\end{array}\right]=\left[\begin{array}{cc}
-\frac{R}{L} & \omega \\
-\omega & -\frac{R}{L}
\end{array}\right]\left[\begin{array}{l}
\hat{i}_{d} \\
\hat{i}_{q}
\end{array}\right]+\left[\begin{array}{c}
\frac{U_{o} \sin \delta_{o}}{L} \\
-\frac{U_{o} \cos \delta_{o}}{L}
\end{array}\right] \hat{\delta}+\left[-\frac{\cos \delta_{o}}{L}\right] \hat{U}_{2}+\left[\begin{array}{c}
\frac{1}{L} \\
L \\
0
\end{array}\right] \hat{U}_{1} \\
{\left[\begin{array}{c}
\hat{P} \\
\hat{Q}
\end{array}\right]=\frac{3}{2}\left[\begin{array}{cc}
U_{o} & 0 \\
0 & U_{o}
\end{array}\right]\left[\begin{array}{c}
\hat{i}_{d} \\
\hat{i}_{q}
\end{array}\right]+\frac{3}{2}\left[\begin{array}{cc}
i_{d o} & 0 \\
0 & i_{q o}
\end{array}\right] \hat{U}_{1}}
\end{gathered}
$$




\section{DROOP CONTROL FOR VSC INTERFACED GENERATORS}

Typically, the VSCs at each generation unit are controlled by a cascaded control loop scheme (Pogaku et al., 2007; Bianchi et al., 2011). A general non-linear control scheme for the VSC is presented Fig. 4. The objective of the droop controller is to provide a voltage and frequency reference to the voltage and current control (V\&CC) in accordance with the demanded power by the microgrid. The demanded power is calculated using (7) and (8). The $\mathrm{V} \& \mathrm{CC}$ is composed of a voltage and current regulator working in a $d q 0$ frame, the design of which can be found e.g. in (Yazdani and Iravani, 2010). The PWM block uses a space vector modulation to drive the IGBTs of the VSC. Only local measurements, $I_{o a b c}, U_{1 a b c}, I_{a b c}$ are needed for the control loops. The RES is supposed to be a constant DC voltage source capable to deliver the demanded amount of power.

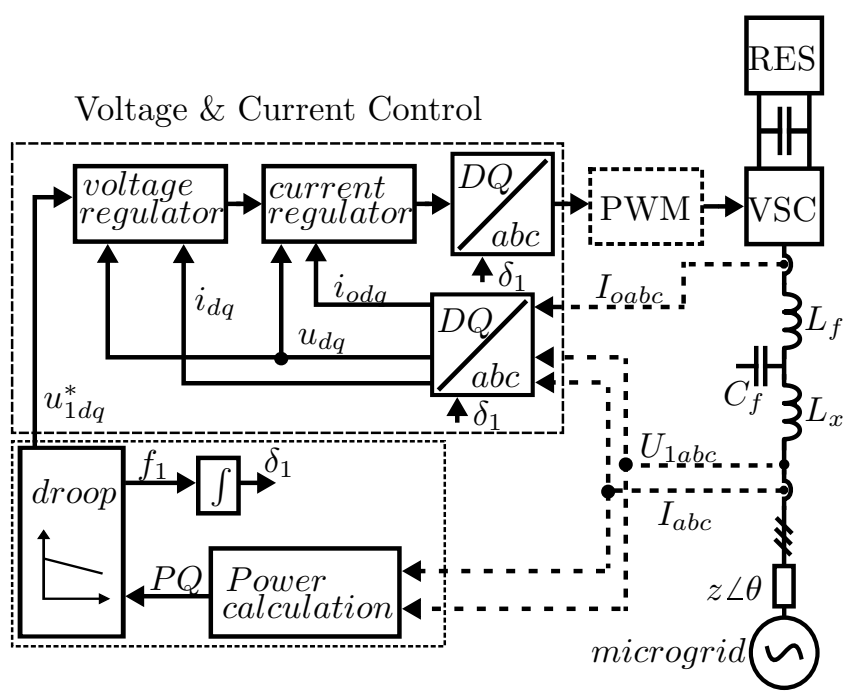

Fig. 4. VSC control scheme

For the design of the droop controller, the cascaded voltage and current control are approximated by a first order system with a time constant $\tau$, and form the $\mathrm{V} \& \mathrm{CC}$ block. The PWM, power devices and filters of the VSC are approximated by an ideal all-pass filter forming the VSC block. The linear model (9)-(10) is used for the power calculation in the equivalent microgrid block in Fig 5 and 6 .

\subsection{Classical droop control}

Commonly the selection of droop coefficients is based on electrical characteristics, rather than for stability reasons. For instance, IEEE Standards Coordinating Committee 21 (2011) suggests to compute the droop coefficients for each generator as

$$
\begin{aligned}
k_{p} & =\frac{f_{\max }-f_{\min }}{P_{\max }}, \\
k_{q} & =\frac{U_{1 \max }-U_{1 \min }}{Q_{\max }} .
\end{aligned}
$$

Classical droop control includes a low-pass filter besides the proportional controller to allow a separation between the power and the voltage and current control loops and to achieve a good power quality injection (Mohamed, 2008; Pogaku et al., 2007). Therefore the classical droop control is implemented as

$$
\begin{aligned}
f^{*}-f_{0} & =-\frac{k_{p}}{\tau_{c} s+1}\left(P-P_{0}\right), \\
U^{*}-U_{0} & =-\frac{k_{q}}{\tau_{c} s+1}\left(Q-Q_{0}\right),
\end{aligned}
$$

where $f_{0}$ and $U_{0}$ are the nominal values for frequency and voltage respectively, $P_{0}$ and $Q_{0}$ are the active and reactive idle power. These last values correspond to the power delivered when the VSC is working at its nominal frequency and voltage, i.e, when the microgrid is connected to utility. These setpoints can be also set by a higher level supervisor control. The droop control scheme is shown in Fig. 5.

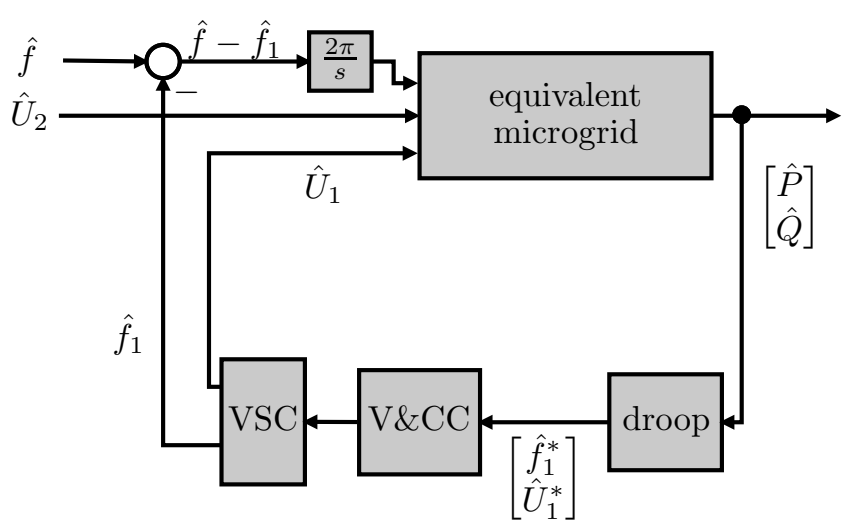

Fig. 5. Linear block diagram of the droop control scheme

The drawbacks of the classical droop control is that the stability is not taken into account during the design. The droop coefficients (11) and (12) producing a good tradeoff between power balancing and power quality might be unable to ensure stability because they do not have enough degrees of freedom as they are just simple proportional controllers.

\subsection{Proposed droop control}

In order to circumvent the stability problems of the classical approach, we propose a droop control scheme based on $H_{\infty}$ optimal control. The main idea is to use the parametrization of the $H_{\infty}$ controllers to decouple the droop coefficient selection from the system stability.

The $H_{\infty}$ optimal control (based on the solution of two Riccati equations (Zhou and Doyle, 1998)) produces a controller such that the $\infty$-norm of the closed loop transfer function between the disturbance $w$ and the performance output $z$ is lower than $\gamma$, i.e. $\left\|T_{z w}\right\|_{\infty}<\gamma$. This controller can be parametrized as

$$
K(s)=J_{11}(s)+J_{12}(s) Q(s)\left(1-J_{22}(s) Q(s)\right)^{-1} J_{21}(s)
$$

where

$$
J(s)=\left[\begin{array}{ll}
J_{11}(s) & J_{12}(s) \\
J_{21}(s) & J_{22}(s)
\end{array}\right]
$$

and $Q(s)$ is a free stable contraction map such that $\|Q(s)\|_{\infty}<\gamma$. Then, by imposing that

$$
K(0)=K_{\text {droop }}=\left[\begin{array}{cc}
k_{p} & 0 \\
0 & k_{q}
\end{array}\right] \text {, }
$$


it can be found the contraction map

$Q(s)=Q(0)=\left(J_{21}(0)\left(K(0)-J_{11}(0)\right)^{-1} J_{12}(0)+J_{22}(0)\right)^{-1}$. As $Q(0)$ is stable, if $\|Q(0)\|<\gamma$ then the desired performance and also the desired power sharing imposed by the droop coefficients $K_{\text {droop }}$ are ensured.

The controller design is cast as a mixed sensitivity problem, with the scheme shown in Fig. 6, where the disturbance $w$ are the frequency $\hat{f}$ and the voltage $\hat{U}_{2}$ and the performance output $z$ is the active and reactive power errors $\bar{P}=\hat{P}-P_{0}$ and $\bar{Q}=\hat{Q}-Q_{0}$ and the control actions, i.e. the voltage reference $\hat{U}_{1}^{*}$ and the frequency reference $\hat{f}_{1}^{*}$ applied to the low level controllers (see Fig. 4). That is,

$$
w=\left[\begin{array}{ll}
\hat{f} & \hat{U}_{2}
\end{array}\right]^{T}, \quad z=\left[\begin{array}{llll}
\bar{P} & \bar{Q} & \hat{f}_{1}^{*} & \hat{U}_{1}^{*}
\end{array}\right]^{T} .
$$

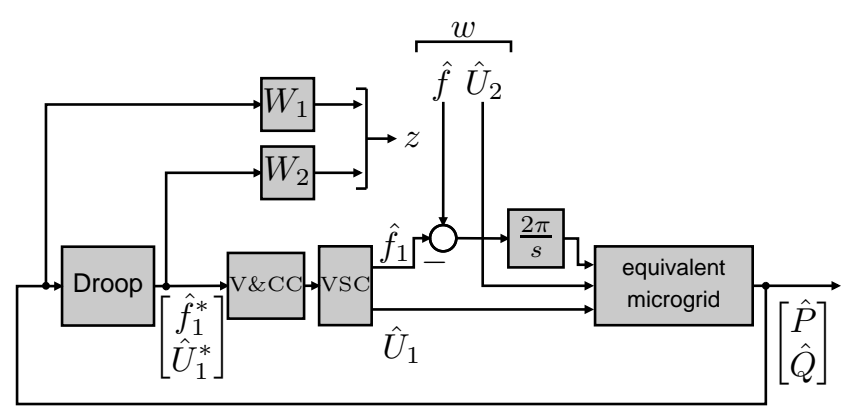

Fig. 6. Setup for the $H_{\infty}$ controller design

As usual, the powers and the control action are affected by weighting functions $W_{1}(s)$ and $W_{2}(s)$, respectively, to obtain the appropriate desirable time response. The weight $W_{1}(s)$ emphasizes the low frequencies components of $\bar{P}$ and $\bar{Q}$ to obtain the desired power errors in low frequencies. Similarly, the function $W_{2}(s)$ penalizes the high frequencies of the controller output in order to avoid unreasonable control actions. A particular choice of these weighing functions is

$$
\begin{aligned}
& W_{1}(s)=\operatorname{diag}\left(10 \frac{s / 10 \omega_{1}+1}{s / 0.001 \omega_{1}+1}, 0.5 \frac{s / 10 \omega_{2}+1}{s / 0.001 \omega_{2}+1}\right), \\
& W_{2}(s)=\frac{s / 0.1 \omega_{3}+1}{s / 100 \omega_{3}+1} K_{\text {droop }}
\end{aligned}
$$

where $\omega_{1}, \omega_{2}$ and $\omega_{3}$ are design parameters.

\section{SIMULATION RESULTS}

This section illustrates the application of the droop design procedure proposed in the previous section. The system under study is shown in Fig. 7 with the parameter values listed in Table 1 . The system include a equivalent migrogrid modelled with an equivalent impedance $R+j \omega L$ and a stiff $\mathrm{AC}$ voltage. Notice that only local measurements and no communications are required for the control loops.

Fig. 8 shows the weighting functions $W_{1}(s)$ and $W_{2}(s)$ used to obtain the droop gains $k_{p}=0.4 \mathrm{~Hz} / \mathrm{kW}$ and $k_{q}=$ $1 \mathrm{~V} / \mathrm{kVar}$, in order to achieve a deviation at full power of $8 \%$ in frequency and $2.5 \%$ in voltage. The response of the system with the optimal controller can be seen in Fig. 9. At time $t=0.35 \mathrm{~s}$ the equivalent microgrid voltage

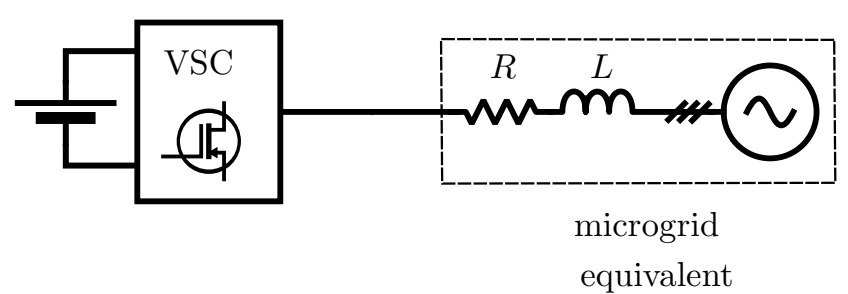

Fig. 7. Microgrid example used to illustrate the proposed droop design

source decreases the frequency in $0.3 \mathrm{~Hz}$. It can be seen that the frequency rapidly reaches the new reference value. The active power $P$ also rapidly converges to the value determined by the droop coefficient.

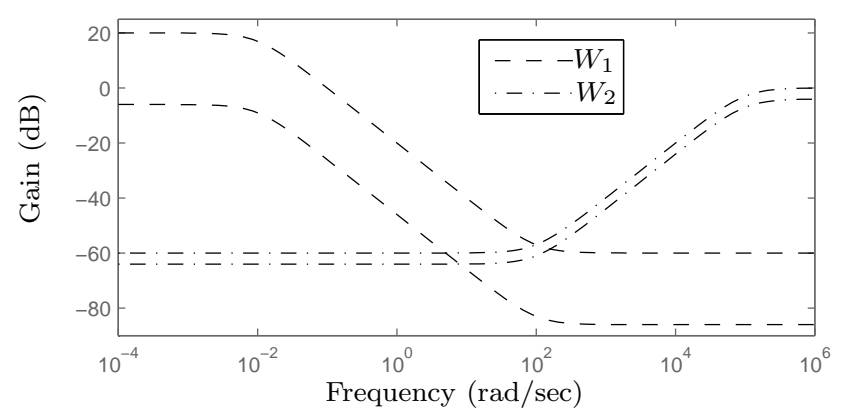

Fig. 8. Typical weighting functions for the mixed sensitivity problem in Fig. 6

If the previous droop coefficients are used in the classical scheme, the system becomes unstable. This fact is evident from Fig. 10, in which the closed loop eigenvalues location are indicated with black dots. With the classical droop, the highest achievable values are around $2.5 \mathrm{~Hz} / \mathrm{kW}$ for active power and $3 \mathrm{~V} / \mathrm{kVar}$ for reactive power.

Table 1. Parameters of the microgrid example

\begin{tabular}{ll}
\hline System voltage: & $400 \mathrm{~V}_{l-l, r m s}, 50 \mathrm{~Hz}$ \\
Line Resistance: & $19.9 \mathrm{~m} \Omega$ \\
Line Inductance: & $0.63 \mathrm{mH}$ \\
VSC PWM filter inductance: & $12.7 \mathrm{mH}$ \\
VSC PWM filter capacitance: & $2.98 \mu \mathrm{F}$ \\
VSC coupling inductance: & $0.12 \mathrm{mH}$ \\
$K_{p}:$ & $0.1 \mathrm{~Hz} / \mathrm{kW}$ \\
$K_{q}:$ & $1 \mathrm{~V} / \mathrm{kVar}$ \\
Switching frequency: & $20 \mathrm{kHz}$ \\
DC voltage of inverter: & $800 \mathrm{~V}$ \\
DC capacitance: & $1020 \mu \mathrm{F}$ \\
\hline
\end{tabular}

To compare the new controller with the classical approach smaller gains of $k_{p}=0.1 \mathrm{~Hz} / \mathrm{kW}$ and $k_{q}=1 \mathrm{~V} / \mathrm{kVar}$ have been chosen. The simulation evaluates the transient and long term behaviour. The droop coefficients are chosen such that the maximum frequency deviation is $2 \%$ form the nominal $50 \mathrm{~Hz}$, and the maximum voltage deviation is $2.5 \%$ from the nominal $400 \mathrm{~V}_{r m s}$. With the aim of a clearer explanation the long term scenario is decomposed into two cases as follows:

Case $1(t \in[0.3,0.7] \mathrm{s}):$ A frequency change of $0.3 \mathrm{~Hz}$ at $t=0.35 \mathrm{~s}$ is imposed by the equivalent microgrid.

Case $2(t \in[0.7,1.0] \mathrm{s})$ : An increment of $1 \mathrm{~kW}$ in the idle power $P_{0}$ (see equation 13 ) at $t=0.8 \mathrm{~s}$. 

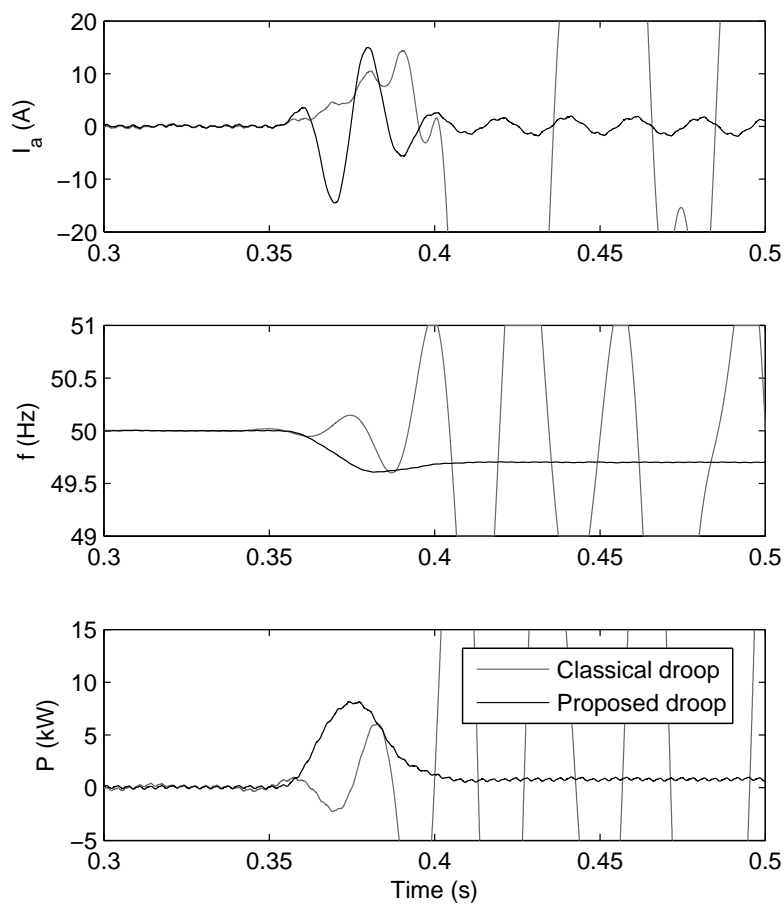

Fig. 9. Response to a change of $0.3 \mathrm{~Hz}$ in grid frequency. In vertical order, plots show the phase $a$ of output current $I_{a}$, frequency $f$, active power $P$ and reactive power $Q$. It can be seen that the system with the classical droop controller approach is unstable

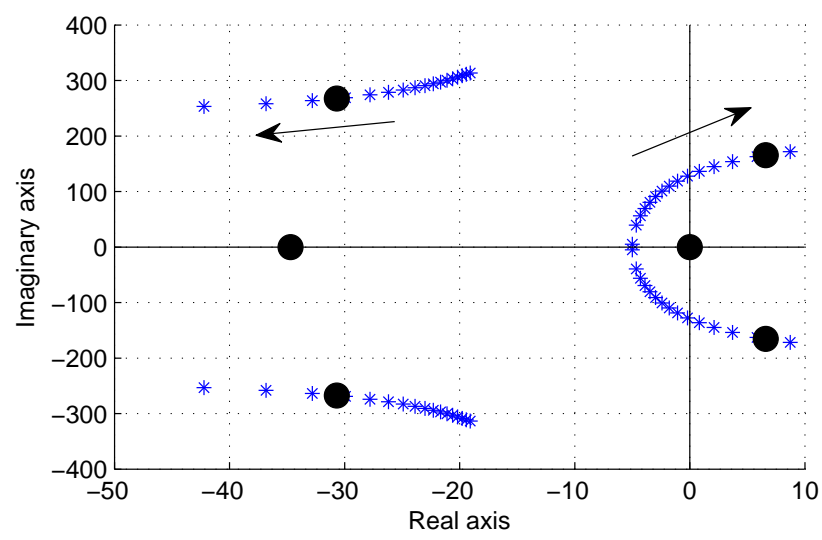

Fig. 10. Eigenvalue analysis using classical droop controller for increasing values of $k_{p}$. Black dots show eigenvalues for $k_{p}=0.4 \mathrm{~Hz} / \mathrm{kW}$ and $k_{q}=1 \mathrm{~V} / \mathrm{kVar}$

\subsection{Case 1: Change in load conditions of the microgrid}

In this case, a change in the load conditions is simulated with a change in the frequency of the $\mathrm{AC}$ voltage source modelling the rest of the microgrid. The frequency disturbance is a gradual change from $50 \mathrm{~Hz}$ to $49.7 \mathrm{~Hz}$ applied at $t_{i}=0.35 \mathrm{~s}$ and ended at $t_{f}=0.37 \mathrm{~s}$. Fig. 11 shows the delivered active power $P$ and reactive power $Q$, phase $a$ of the output current $I$ and frequency $f$ produced by the converter. The dark lines corresponds to the system with proposed control and the grey lines to the system with the classical approach.

Observe that the proposed control achieves a more damped response than the classical scheme. All the variables cor-
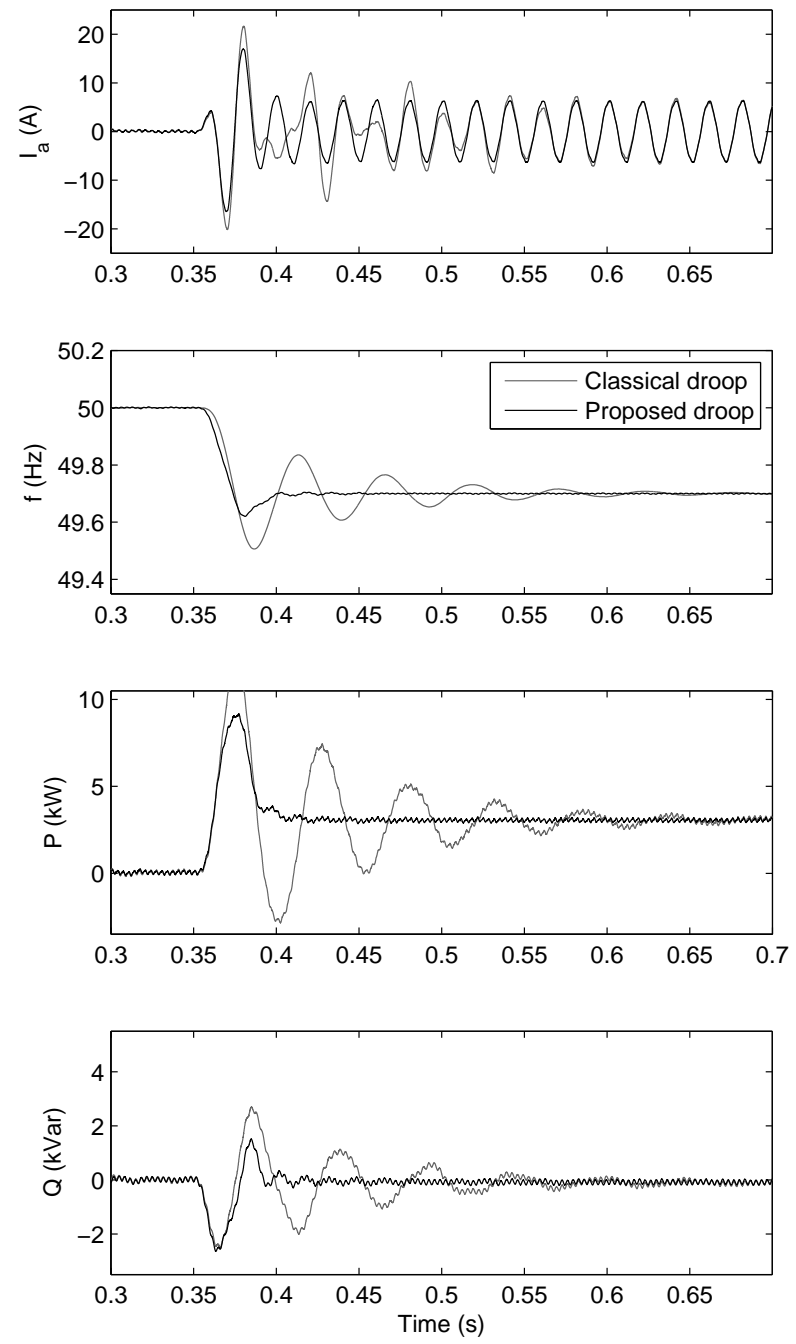

Fig. 11. Case 1: Response to a change of $0.3 \mathrm{~Hz}$ in grid frequency. In vertical order, plots show the phase $a$ of the output current $I$, frequency $f$, active power $P$ and reactive power $Q$

responding to proposed droop in Fig. 11 settle rapidly to the new equilibrium values, whereas the system with the classical droop needs much more time to converge to the final values. Notice also that the small oscillations in the proposed and classical approach are caused by the switching of the IGBTs.

\subsection{Case 2: Change in active idle power reference}

In the scenario presented in Fig. 12 the droop controller setpoints are changed, as it would be done by a supervisor or higher level control layer (De Brabandere et al., 2007). In this case, the active idle power setpoint is shifted from $0 \mathrm{~kW}$ to $1 \mathrm{~kW}$ at $t=0.8 \mathrm{~s}$. The responses in Fig. 11 follows the ones in Fig. 11, as they belong to the same simulation of one second. The effectiveness of the proposed control is more noticeable in the frequency and the active power. Both the frequency and the power in the case of the proposed approach converge rapidly to the new equilibrium, while the classical droop presents more oscillatory behaviour. 

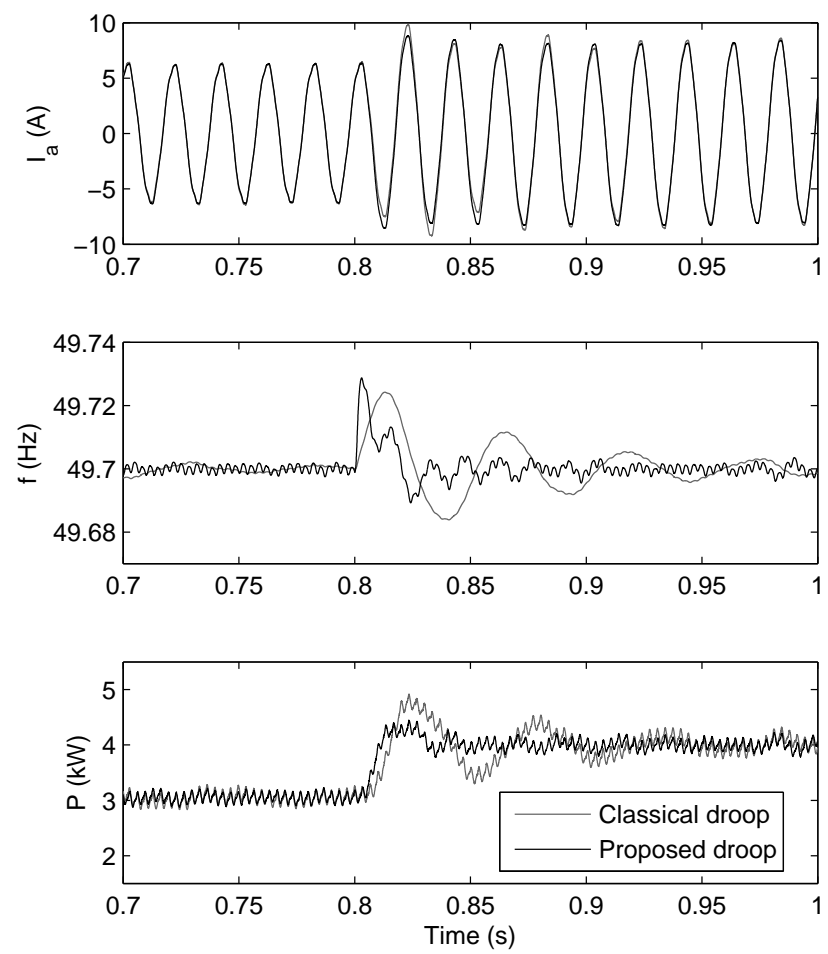

Fig. 12. Case 2: Response to a increment of $1 \mathrm{~kW}$ in active idle power. In vertical order, plots show the phase $a$ of the output current $I$, frequency $f$ and active power $P$

\section{CONCLUSIONS}

In this paper, a new droop control approach for RES with VSC interface forming islanded microgrids has been proposed. The new control design procedure permits to decouple the selection of the droop, to achieve the desire power sharing, from the problem of ensuring closed loop stability. The proposed procedure is based on the parametrization of the $H_{\infty}$ optimal controllers which allows to set the DC gain of the controller according to the droop coefficient given by the power sharing. Compared with classical approaches, the new scheme provides additional degrees of freedom to obtain a faster convergence of the variables to the steady state values. These conclusions have been illustrated by simulation under several scenarios using a model including switching of the IGBT of the VSC.

\section{REFERENCES}

Agrawal, M. and Mittal, A. (2011). Micro Grid Technological Activities Across the Globe . International Journal of Research and Reviews in Applied Sciences, 7(2), 147152.

Akagi, H., Watanabe, E.H., and Aredes, M. (2007). Instantaneous Power Theory and Applications to Power Conditioning. Wiley.

Allen, E. and Ilic, M. (2000). Interaction of transmission network and load phasor dynamics in electric power systems. Circuits and Systems I: Fundamental Theory and Applications, IEEE Transactions on, 47(11), 1613 -1620 .

Bianchi, F., Gomis-Bellmunt, O., Egea-Alvarez, A., and Junyent-Ferre, A. (2011). Optimal control of voltage source converters for fault operation. In Power Electronics and Applications (EPE 2011), Proceedings of the 2011-14th European Conference on, 1-10.

De Brabandere, K., Vanthournout, K., Driesen, J., Deconinck, G., and Belmans, R. (2007). Control of microgrids. In Power Engineering Society General Meeting, $200 \%$. IEEE, 1-7. IEEE.

EU Commission (2006). Vision and strategy for european electricity networks of the future. Technical report, EU commission, climate action.

EU Commission (2010). EU climate and energy package. Technical report, EU commission, climate action.

Guerrero, J., de Vicuna, L., Matas, J., Castilla, M., and Miret, J. (2004). A wireless controller to enhance dynamic performance of parallel inverters in distributed generation systems. Power Electronics, IEEE Transactions on, 19(5), 1205 - 1213. doi: 10.1109/TPEL.2004.833451.

IEEE Standards Coordinating Committee 21 (2011). IEEE guide for design, operation, and integration of distributed resource island systems with electric power systems. IEEE Std 1547.4-2011, 1 -54.

Lasseter, R. (2002). Microgrids. In Power Engineering Society Winter Meeting, 2002. IEEE, volume 1, $305-$ 308 vol.1. doi:10.1109/PESW.2002.985003.

Lopes, J. and Moreira, C. (2006). Defining control strategies for microgrids islanded operation. Power Systems, IEEE, 21(2), 916-924.

Mohamed, Y. (2008). Adaptive decentralized droop controller to preserve power sharing stability of paralleled inverters in distributed generation microgrids. Power Electronics, IEEE, 23(6), 2806-2816.

Pogaku, N., Prodanovic, M., and Green, T. (2007). Modeling, analysis and testing of autonomous operation of an inverter-based microgrid. Power Electronics, IEEE Transactions on, 22(2), $613-625$.

Roman-Barri, M., Cairo-Molins, I., Sumper, A., and Sudria-Andreu, A. (2010). Experience on the implementation of a microgrid project in barcelona. In Innovative Smart Grid Technologies Conference Europe (ISGT Europe), 2010 IEEE PES, 1 -7. doi: 10.1109/ISGTEUROPE.2010.5638996.

Venkatasubramanian, V., Schattler, H., and Zaborszky, J. (1995). Fast time-varying phasor analysis in the balanced three-phase large electric power system. $A u$ tomatic Control, IEEE Transactions on, 40(11), 1975 $-1982$.

Yazdani, A. and Iravani, R. (2010). Voltage-sourced converters in power systems. Wiley.

Zhou, K. and Doyle, J.C. (1998). Essentials of robust control. Prentice Hall. 